\title{
Cloud-Chamber Study of Negative Strange Particles*
}

\author{
G. H. Trilling $\dagger$ and G. Neugebauer \\ California Institute of Technology, Pasadena, California
}

(Received September 11, 1956)

A detailed analysis of the decays in flight of negative $K$ mesons and hyperons, obtained with the 48-in. cloud chambers in Pasadena, is presented. The principal results are the following:

1. Two new fully measurable $\tau^{-}$events add to the already strong evidence for the existence of this particle.

2. $K^{-}$events with a single charged secondary are shown to be most consistent with the $K_{\pi 2}$ and $K_{\mu 2}$ decay modes.

3. An analysis of 7 Caltech $\Xi^{-}$events is shown to lead to a $Q$ value of $65 \pm 3 \mathrm{Mev}$, and a $50 \%$ confidence lower limit to the lifetime of $4.6 \times 10^{-10} \mathrm{sec}$. A rough upper limit to the lifetime of $2 \times 10^{-8} \mathrm{sec}$, based on the predictions of the strangeness theory, is obtained. Angular correlations among the $\Xi^{-}$secondaries are studied, but the statistics are insufficient to permit any significant conclusion. An additional event which is most easily interpreted as a $\Xi^{-}$decay is discussed.

4. The $V^{-}$events which are neither $K^{-}$nor $\Xi^{-}$are shown to have a lifetime less than or equal to $\left(1.52_{-0.25}{ }^{+0.38}\right) \times 10^{-10} \mathrm{sec}$ and a $P^{*}$ value, based on their transverse momentum distribution, of $197 \pm 7 \mathrm{Mev} / c$. These values correspond satisfactorily with the presently known properties of the $\boldsymbol{\Sigma}^{-}$hyperon.

5. It is estimated from a comparison of the numbers of identified $\Xi^{-}$'s and $V^{- \text {'s }}$ that the fraction of $\Lambda^{0}$ particles which decay into charged secondaries is greater than $0.40_{-0.11}^{+0.15}$, and can, insofar as the present data are concerned, be as high as unity.

All errors quoted in the above results are $50 \%$ confidence limits.

\section{INTRODUCTION}

$\mathrm{K}^{\mathrm{N}}$ NOWLEDGE of the properties of positively charged hyperons and heavy mesons has been greatly increased recently as a result of the production of these particles under highly controllable conditions in large accelerators and the analysis of their decays at rest in photographic emulsion stacks. ${ }^{1}$ Indeed the great precision possible in this way has diminished the value derived from studies of the decays of positively charged $V$ particles in cloud chambers.

On the other hand, because negative mesons and hyperons brought to rest in emulsions interact rather than decay, information on their decay schemes and other properties must be obtained from observations of decays in flight. For this reason, magnetic-cloudchamber studies of negative particles can contribute important information.

The data presented in this paper were obtained with the 48-in. magnet cloud chambers in Pasadena. During $3 \frac{1}{2}$ years of operation, $3 \tau^{-}$events and $115 V^{-}$events were obtained out of about 50000 photographs. Although some discussion of a part of these events has been given in earlier reports, ${ }^{2,3}$ the present paper provides a more detailed analysis of those properties of negative unstable particles concerning which present knowledge is still fragmentary.

\section{II. $K^{-}$MESONS}

\section{A. $\tau^{- \text {Events }}$}

$\tau$ mesons are easily recognized in cloud chambers by their decay into three charged secondaries. The first

* Assisted by the joint program of the Office of Naval Research and the U. S. Atomic Energy Commission.

$\dagger$ Now at the Ecole Polytechnique, Paris, France.

1 Ritson, Pevsner, Fung, Widgoff, Zorn, Goldhaber, and Goldhaber, Phys. Rev. 101, 1085 (1956).

${ }^{2}$ G. H. Trilling and R. B. Leighton, Phys. Rev. 100, 1468 (1955).

${ }^{3}$ Sorrels, Leighton, and Anderson, Phys. Rev. 100, 1457 (1955). identified $\tau^{-}$event was obtained several years ago with the 48 -in. cloud chambers. ${ }^{4}$ Because few other such events have been reported, ${ }^{5}$ and because the momentum and angular distributions of the secondaries are of interest in determining the spin and parity relations of the $\tau$ meson, ${ }^{6}$ it is perhaps worthwhile to present data on two cases recently obtained.

The data on these events are summarized in Table I. In addition to the presence of the three charged secondaries, other evidence for the correctness of the identification of these events may be summarized as follows:

1. Momentum and ionization estimates on the secondaries set upper limits on their masses which are inconsistent with the mass of any known particle heavier than a $\pi$ meson. The positive secondary of event 64388 can be definitely identified from the fact that it undergoes $\pi-\mu$ decay.

2 . The $Q$ values are in satisfactory agreement with the accepted value of $75.0 \pm 0.8 \mathrm{Mev}^{7}$

TABLE I. Data on two $\tau^{-}$decay events $(P=$ laboratory momentum, $I=$ ionization, $(\Delta x, \Delta y, \Delta z)=$ components of the tangent to each track at the point of decay ${ }^{\mathfrak{a}}$.

\begin{tabular}{|c|c|c|c|c|c|c|c|}
\hline $\begin{array}{l}\text { Case } \\
\text { No. }\end{array}$ & $\begin{array}{l}\text { Par- } \\
\text { ticle }\end{array}$ & $\underset{(\mathrm{Mev} / c)}{P}$ & $I$ & $\begin{array}{c}\Delta x \\
(\mathrm{~cm})\end{array}$ & $\begin{array}{c}\Delta y \\
(\mathrm{~cm})\end{array}$ & $\underset{(\mathrm{cm})}{\Delta z}$ & $\begin{array}{c}Q \\
(\mathrm{Mev})\end{array}$ \\
\hline 63325 & $\begin{array}{l}\tau^{-} \\
\pi^{+} \\
\pi^{-} \\
\pi^{-}\end{array}$ & $\begin{array}{l}789_{-67}+91 b \\
152^{-14} \\
370_{-55}^{+77} \\
294_{-35}^{+46}\end{array}$ & $\begin{array}{l}<2 \\
<2 \\
<2 \\
<2\end{array}$ & $\begin{array}{l}3.13 \\
2.52 \\
8.74 \\
6.76\end{array}$ & $\begin{array}{r}-5.24 \\
-9.89 \\
-10.81 \\
-10.97\end{array}$ & $\begin{array}{r}-0.18 \\
2.85 \\
0.11 \\
-3.99\end{array}$ & $69_{-11}+16$ \\
\hline 64388 & $\begin{array}{l}\tau^{-} \\
\pi^{+} \\
\pi^{-} \\
\pi^{-}\end{array}$ & $\begin{array}{c}323 \pm 28 b \\
39 \pm 3 \\
223 \pm 23 \\
142 \pm 15\end{array}$ & $\begin{array}{c}2-3.5 \\
5-10 \\
<2 \\
1.3-2.5\end{array}$ & $\begin{array}{r}1.21 \\
7.10 \\
8.09 \\
-1.99\end{array}$ & $\begin{array}{r}-4.62 \\
-18.96 \\
-14.62 \\
-9.64\end{array}$ & $\begin{array}{r}-0.58 \\
-1.98 \\
7.00 \\
-11.28\end{array}$ & $95 \pm 13$ \\
\hline
\end{tabular}

a The errors in the measurements of $\Delta x$ and $\Delta y$ are $\pm 0.05 \mathrm{~cm}$ and, in the measurement of $\Delta z, \pm 0.15 \mathrm{~cm}$.

$\mathrm{b}$ The momenta of the primaries were computed by taking the vector sum of the secondary momenta.

${ }^{4}$ V. A. J. van Lint and G. H. Trilling, Phys. Rev. 92, 1089 (1953).

${ }_{5}$ Aggson, Fretter, Friesen, Hansen, and Lagarrigue, Phys. Rev. 102, 243 (1956).

${ }^{6}$ R. Dalitz, Phys. Rev. 94, 1046 (1954).

7 A. M. Shapiro, Revs. Modern Phys. 28, 164 (1956). 
TABLE II. Data on five $K_{1}^{-}$events for which an accurate $P^{*}$ value can be obtained $\left(P_{1}=\right.$ momentum of primary, $I_{1}=$ ionization of primary, $P_{2}=$ momentum of secondary, $I_{2}=$ ionization of secondary, $\theta=$ angle of decay, $P^{*}(\mu)=$ center-of-mass momentum assuming $\mu$-meson secondary, $P^{*}(\pi)=$ center-of-mass momentum assuming $\pi$-meson secondary, $\Delta P^{*}=$ probable error in $\left.P^{*}\right)$.

\begin{tabular}{|c|c|c|c|c|c|c|c|c|}
\hline $\begin{array}{l}\text { Case } \\
\text { No. }\end{array}$ & $\begin{array}{c}P_{1} \\
(\mathrm{Mev} / c)\end{array}$ & $I_{1}$ & $\begin{array}{c}P_{2} \\
(\mathrm{Mev} / c)\end{array}$ & $I_{2}$ & $\stackrel{\theta}{(\mathrm{deg})}$ & $\begin{array}{c}P^{*}(\mu) \\
(\mathrm{Mev} / c)\end{array}$ & $\begin{array}{c}P^{*}(\pi) \\
(\mathrm{Mev} / c)\end{array}$ & $\begin{array}{c}\Delta P^{*} \\
(\mathrm{Mev} / c)\end{array}$ \\
\hline 24018 & $101 \pm 13$ & $10-20$ & $220 \pm 55$ & $<2$ & 102 & 236 & 238 & \pm 58 \\
\hline 26717 & $204_{-62}^{+125}$ & $2-4$ & $280 \pm 65$ & $1.3-2$ & 41.5 & 194 & 190 & \pm 45 \\
\hline 52791 & $725 \pm 140$ & $<2$ & $637 \pm 60$ & $<2$ & 19 & 243 & 236 & \pm 32 \\
\hline 64559 & $360 \pm 60$ & $1.5-3$ & $425 \pm 42$ & $<2$ & 11 & 210 & 207 & \pm 31 \\
\hline 46944 & $515 \pm 45$ & $<2$ & $205 \pm 17$ & $<2$ & 60 & 200 & 208 & \pm 17 \\
\hline
\end{tabular}

3. The transverse momentum balance about the line of flight of the primary is satisfactory for both events.

4. The ionization and momentum of the primary of event 64388 indicate a mass of $730 m_{e}-1350 m_{e}$, in good agreement with the $\tau$ mass.

\section{B. $K_{1}$ - Events}

The symbol $K_{1}$ will be used to denote $K$ events in which only one charged secondary is produced. The $K_{1}{ }^{+}$decay modes have been studied in great detail with photographic emulsions exposed to the $K$-particle beam at the Bevatron. ${ }^{1}$ Some evidence that the $K_{1}-$ modes are symmetric to these has already been obtained from decays in flight observed in Bevatron-exposed photographic emulsions ${ }^{8}$ and in cosmic-ray cloud-chamber observations. $^{9}$

Further evidence in this respect has been obtained in the present work by the analysis of $V^{-}$events whose primaries can be identified as $K$ particles. This identification has been possible for 8 events on the basis of the momentum and ionization of the primaries. Four of these events have secondaries whose momenta are sufficiently measurable to permit determinations of the $P^{*}$ values to accuracies of better than $25 \%$. Data on these events are presented in the first four rows of Table II. For completeness, event 46944 , obtained with the 48 -in. chambers in the course of a separate experiment, ${ }^{10}$ is also listed in Table II. It is clear from inspection of Table II that the events are most easily interpreted as the decays of $K^{-}$mesons by either of the modes :

$$
\begin{array}{ll}
K_{\mu 2^{-} \rightarrow \mu^{-}+\nu} & P^{*}=236 \mathrm{Mev} / c, \\
K_{\pi 2^{-} \rightarrow \pi^{-}+\pi^{0}} & P^{*}=206 \mathrm{Mev} / c .
\end{array}
$$

The $P^{*}$ value of event 46944 strongly suggests the $K_{\pi 2}$ decay mode; in the other cases, the measurement errors are too large to distinguish between the above decay schemes.

One can conclude from these data that the predominant $K_{1}$ - decay modes are two-body decays consistent with either $K_{\mu 2}$ or $K_{\pi 2}$ modes. This conclusion, when

${ }^{8}$ A. G. Ekspong and G. Goldhaber, Phys. Rev. 102, 1187 (1956).

${ }^{9}$ Armenteros, Astier, D'Andlan, Gregory, Mendel, Hennesey, Lagarrigue, Leprince-Ringuet, Mueller, and Peyrou, "Proceedings of the International Conference on Elementary Particles, Pisa, 1955," Nuovo cimento (to be published).

10 The authors are indebted to Dr. Carl Rouse for the use of this event. combined with knowledge of the existence of $\tau^{-}$mesons, gives added weight to the belief that $K^{-}$mesons decay by modes symmetric to those of $K^{+}$mesons.

\section{Plus to Minus Ratios of $K$ Particles}

One of the most striking features observed in studies of $K$ particles, from both cosmic rays and accelerators, is the excess of slow positives over slow negatives. This observation is explained in the Gell-Mann strangeness theory ${ }^{11}$ by the fact that some of the reactions in which $K^{+}$mesons are produced have lower thresholds than those in which $K^{-}$mesons are produced. Indeed, the $43 K_{1}{ }^{+}$and $8 K_{1}^{-}$mesons observed during the course of the present experiment exhibit precisely this behavior. In contrast, the numbers of $\tau$ events observed during the same time are $4 \tau^{+}, 3 \tau^{-}$, and $1 \tau^{\text {? }}$. Furthermore, the mean momentum of the $\tau^{-}$'s is only $484 \mathrm{Mev} / c$ as compared to $856 \mathrm{Mev} / c$ for the $\tau^{+}$'s. If it is assumed that the observed $\tau$ 's and $K_{1}$ 's are drawn from populations having identical proportions of positive and negative particles, the probability of observing an asymmetry between $\tau$ 's and $K_{1}$ 's as great or greater than the one obtained is about $10 \%$. Thus, while a difference between the $\tau$ 's and $K_{1}$ 's seems to be suggested, it cannot yet be considered significant in view of the meager statistics involved.

\section{III. $\Xi^{-}$HYPERONS}

\section{A. General Discussion}

Evidence for the existence of hyperons which decay by the mode

$$
\begin{array}{r}
\Xi^{-} \rightarrow \Lambda^{0}+\pi^{-} \\
\searrow+\pi^{-}
\end{array}
$$

has already been reported. ${ }^{22}$ Cascade decays must satisfy a number of conditions, at least part of which can usually be checked with ease. It is very improbable that events other than $\mathbf{Z}^{-}$decays can satisfy any, let alone all, of these conditions:

1. The apex of the $V^{-}$decay must lie in the plane of the $V^{0}$ decay.

${ }^{11}$ M. Gell-Mann and A. Pais, Proceedings of the 1954 Glasgow Conference on Nuclear and Meson Physics (Pergamon Press, London, 1955).

${ }_{12}$ Armenteros, Baker, Butler, Cachon, and York, Phil. Mag. 43, 597 (1952); Anderson, Cowan, Leighton, and van Lint, Phys. Rev. 92, 1089 (1953); E. W. Cowan, Phys. Rev. 94, 161 (1954). 
2. The apex of the $V^{0}$ decay must lie in the plane of the $V^{-}$decay.

3. The transverse momenta of the $V^{0}$ and $\pi^{-}$about the line of flight of the $V^{-}$primary must balance.

4. The transverse momenta of the $V^{0}$ decay products about the line of flight of the $V^{0}$ primary must balance.

5. The $V^{0}$ decay dynamics must be consistent with those expected for $\Lambda^{0}$ decay. In particular, the quantity $P_{-} \sin \theta_{\text {, where }} P_{-}$is the momentum of the negative secondary of the $V^{0}$ and $\theta$ is the $V^{0}$ opening angle, must be less than $118 \mathrm{Mev} / c$, a requirement which, if the $V^{0}$ were really a $\theta^{0}$, would have only a $10 \%$ chance of being fulfilled. ${ }^{13}$ The value of this test lies in the fact that the strangeness theory forbids the associated production of a $\Lambda^{0}$ and any negative strange particle.

6. Present knowledge on the energy release in $z^{-}$ decay requires that the transverse momentum of the charged secondary of the decay be less than about 140 $\mathrm{Mev} / c$. It may be noted that the maximum transverse momentum of the secondary in $\Sigma^{-}$or $K^{-}$decay is about $200 \mathrm{Mev} / c$.

On the basis of the above conditions, six events exhibiting a $V^{-}$and a $V^{0}$ decay were identified as $\Xi^{-}$ decays. The data from one of these events have already been published in detail. ${ }^{3}$ The results of measurements performed on the other five are shown in Table III. The extent to which these events satisfy the require-

TABLE III. Data on five $\Xi^{-}$events. The particles are labeled as follows: (1) $\Xi^{-}$primary, (2) $\pi^{-}$from $\Xi^{-}$decay, (3) $\Lambda^{0}$ from $\Xi^{-}$ decay, (4) proton from $\Lambda^{0}$ decay, (5) $\pi^{-}$from $\Lambda^{0}$ decay.

\begin{tabular}{|c|c|c|c|c|c|}
\hline $\begin{array}{l}\text { Case } \\
\text { No. }\end{array}$ & $\begin{array}{l}\text { Par- } \\
\text { ticle }\end{array}$ & $\begin{array}{c}P \\
(\mathrm{Mev} / c)\end{array}$ & $\begin{array}{l}\Delta x \\
(\mathrm{~cm})\end{array}$ & $\begin{array}{c}\Delta y \\
(\mathrm{~cm})\end{array}$ & $\underset{(\mathrm{cm})}{\Delta z}$ \\
\hline 09691 & $\begin{array}{l}(1) \\
(2) \\
(3) \\
(4) \\
(5)\end{array}$ & $\begin{array}{c}\ldots \\
192 \pm 25 \\
\ldots \\
\cdots \\
275 \pm 15\end{array}$ & $\begin{array}{r}-2.00 \\
-10.15 \\
-1.62 \\
-1.74 \\
-4.62\end{array}$ & $\begin{array}{l}-5.56 \\
-7.52 \\
-5.44 \\
-7.08 \\
-6.72\end{array}$ & $\begin{array}{l}-1.12 \\
-5.02 \\
-1.22 \\
-1.50 \\
-0.43\end{array}$ \\
\hline 17152 & $\begin{array}{l}(1) \\
(2) \\
(3) \\
(4) \\
(5)\end{array}$ & $\begin{array}{c}\ldots \\
390_{-90}^{+170} \\
\cdots \\
\cdots \\
295 \pm 15\end{array}$ & $\begin{array}{l}1.49 \\
5.23 \\
1.32 \\
0.80 \\
0.06\end{array}$ & $\begin{array}{r}-5.63 \\
-10.09 \\
-6.54 \\
-3.19 \\
-3.34\end{array}$ & $\begin{array}{r}-1.00 \\
-1.75 \\
-1.21 \\
-0.87 \\
0.30\end{array}$ \\
\hline 22850 & $\begin{array}{l}(1) \\
(2) \\
(3) \\
(4) \\
(5)\end{array}$ & $\begin{array}{c}\cdots \\
396 \pm 15 \\
\cdots \\
\cdots \\
230 \pm 35\end{array}$ & $\begin{array}{r}0.92 \\
17.25 \\
1.00 \\
0.61 \\
0.55\end{array}$ & $\begin{array}{r}-8.40 \\
-35.47 \\
-17.86 \\
-13.15 \\
-9.76\end{array}$ & $\begin{array}{r}-1.49 \\
-4.62 \\
-3.27 \\
-3.00 \\
1.02\end{array}$ \\
\hline 44094 & $\begin{array}{l}(1) \\
(2) \\
(3) \\
(4) \\
(5)\end{array}$ & $\begin{array}{c}1750 \pm 450 \\
164 \pm 13 \\
\ldots \\
\ldots \\
\ldots\end{array}$ & $\begin{array}{r}-1.60 \\
7.22 \\
-1.66 \\
-0.51 \\
-0.92\end{array}$ & $\begin{array}{r}-17.72 \\
-12.28 \\
-10.78 \\
-3.80 \\
-3.40\end{array}$ & $\begin{array}{r}-1.72 \\
2.38 \\
-1.45 \\
-0.38 \\
-0.20\end{array}$ \\
\hline 58274 & $\begin{array}{l}(1) \\
(2) \\
(3) \\
(4) \\
(5)\end{array}$ & $\begin{array}{c}\ddot{3} \\
454 \pm 40 \\
\cdots \\
\ddot{*} \\
365 \pm 60\end{array}$ & $\begin{array}{r}0.57 \\
23.70 \\
3.98 \\
2.66 \\
8.03\end{array}$ & $\begin{array}{r}-3.00 \\
-40.40 \\
-20.54 \\
-16.47 \\
-18.31\end{array}$ & $\begin{array}{l}0.57 \\
5.85 \\
3.45 \\
2.85 \\
2.87\end{array}$ \\
\hline
\end{tabular}

${ }^{13}$ J. P. Astbury, Nuovo cimento 12, 387 (1954). ments for $\boldsymbol{\Xi}^{-}$identification is shown in Table IV. Those momenta needed for the check on transverse momentum balance, which could not be measured directly, were computed by using the known $\Lambda^{0} Q$ value.

\section{B. $Q$ Value of $\Xi^{-}$Events}

The $Q$ values for the Caltech $\Xi^{-}$events, computed from the measured momenta and angles and the known $\Lambda^{0} Q$-value, are listed in Table $\mathrm{V}$. The weighted mean is $65 \pm 3 \mathrm{Mev}$ corresponding to a mass value for the $\Xi^{-}$of $(2582 \pm 6) m_{e}$; the quoted errors are probable errors.

\section{Lifetime of $\Xi^{-}$Events}

Unfortunately the $\Xi^{-}$events do not lend themselves to accurate lifetime analysis for two reasons:

1. The number of events available is small.

2. The necessity of seeing the decay of the $\Lambda^{0}$ to identify the $\Xi^{-}$reduces the gate time determined by the chamber geometry by an amount of the order of the $\Lambda^{0}$ lifetime.

Despite these limitations, it is possible to obtain a useful lower limit to $\tau_{\Xi}$. The usual maximum likelihood procedure may be used, ${ }^{14}$ provided that the likelihood function for each event includes not only the decay probability of the $\Xi^{-}$but also the decay probability of the $\Lambda^{0}$.

The most convenient way of treating the data is to calculate the $S$ function, defined by the relation

$$
S \equiv \frac{\partial \ln L / \partial \tau_{\Xi}}{\left\{\left\langle-\partial^{2} \ln L / \partial \tau \Xi^{2}\right\rangle\right\}^{\frac{1}{2}}}=\frac{\Sigma_{i} t_{i}-\Sigma_{i}\left\langle t_{i}\right\rangle}{\left\{\Sigma_{i}\left\langle t_{i}{ }^{2}\right\rangle-\Sigma_{i}\left\langle t_{i}\right\rangle^{2}\right\}^{\frac{1}{2}}},
$$

where $L$ is the combined likelihood function for all the events, $\tau_{\Xi}$ is the $\Xi^{-}$lifetime, $t_{i}$ is the observed time of flight of each $\Xi^{-}$and the angular brackets $(\langle\rangle)$denote averages over all possible $a$ prior $i$ values of $t_{i}$ for a given lifetime $\tau_{\Xi}$ and the observed gate time. If the correct value of $\tau \approx$ is used, $S$ has zero mean, unit variance, and an approximately normal distribution. Conversely, for a given set of $t_{i}$ 's the value $S=0$ corresponds to the most probable value of $\tau \Xi$, and the probability that a given lifetime $\tau_{\Xi}$ gave rise to the measured times of flight

TABLE IV. Evidence for ${ }^{-}$-identification $\left(\delta_{1}=\right.$ angle between $V^{0}$ decay plane and assumed $V^{0}$ line of flight, $\delta_{2}=$ angle between $V^{-}$ decay plane and assumed $V^{0}$ line of flight, $\Delta_{1}=$ unbalance in the transverse momenta of the $V^{-}$secondaries about the $V^{-}$line of flight, $\Delta_{2}=$ unbalance in the transverse momenta of the $V^{0}$ secondaries about the assumed $V^{0}$ line of flight, $P_{-}=$momentum of negative secondary of $V^{0}, \theta=V^{0}$ opening angle, $\bar{P}_{T}=$ transverse momentum of charged secondary of $V^{-}$about $V^{-}$line of flight).

\begin{tabular}{|c|c|c|c|c|c|c|}
\hline $\begin{array}{l}\text { Case } \\
\text { No. }\end{array}$ & $\begin{array}{c}\delta_{1} \\
(\mathrm{deg})\end{array}$ & $\begin{array}{c}\delta_{2} \\
(\operatorname{deg})\end{array}$ & $\left(\begin{array}{c}\Delta_{1} \\
\text { ev } / c)\end{array}\right.$ & $\left(\begin{array}{c}\Delta_{2} \\
(\mathrm{ev} / c)\end{array}\right.$ & $\begin{array}{l}P-\sin \theta \\
(\mathrm{Mev} / c)\end{array}$ & $\begin{array}{c}P T \\
(\mathrm{Mev} / c)\end{array}$ \\
\hline $\begin{array}{l}09691 \\
17152 \\
22850 \\
44094 \\
58724\end{array}$ & $\begin{array}{l}1.4 \pm 2.9 \\
0.4 \pm 1.5 \\
0.5 \pm 0.5 \\
2.2 \pm 3.0 \\
0.1 \pm 0.7\end{array}$ & $\begin{array}{l}2.5 \pm 3.5 \\
0.2 \pm 2.0 \\
0 \pm 0.8 \\
0.5 \pm 1.0 \\
1.1 \pm 4.0\end{array}$ & $\begin{array}{c}25 \pm 63 \\
14 \pm 42 \\
0 \pm 30 \\
14 \pm 40 \\
83 \pm 220\end{array}$ & $\begin{array}{r}0 \pm 41 \\
33 \pm 96 \\
38 \pm 40 \\
18 \pm 28\end{array}$ & $\begin{array}{r}107 \pm 7 \\
119 \pm 13 \\
74 \pm 11 \\
91 \pm 17\end{array}$ & $\begin{array}{c}108 \pm 15 \\
84 \pm 26 \\
133 \pm 6 \\
102 \pm 8 \\
154 \pm 35\end{array}$ \\
\hline
\end{tabular}

${ }^{14}$ M. S. Bartlett, Phil. Mag. 44, 249 (1953). 
TABLE V. Properties of $\Xi^{-}$events.

\begin{tabular}{ccccc}
\hline \hline $\begin{array}{c}\text { Case } \\
\text { No. }\end{array}$ & $\begin{array}{c}Q \\
(\mathrm{Mev})\end{array}$ & $\begin{array}{c}t_{i} \\
\left(10^{-10} \mathrm{sec}\right)\end{array}$ & $\begin{array}{c}T_{i} \\
\left(10^{-10} \mathrm{sec}\right)\end{array}$ & $\begin{array}{c}\Theta \\
(\mathrm{deg})\end{array}$ \\
\hline 04559 & $\ldots$ & 1.2 & 2.6 & $\ldots$ \\
09691 & $72 \pm 8$ & 0.6 & 2.6 & 49 \\
17152 & $45 \pm 16$ & 1.4 & 3.1 & 78 \\
22850 & $63 \pm 4$ & 1.1 & 4.3 & 77 \\
44094 & $52 \pm 13$ & 5.8 & 8.2 & 50 \\
58274 & $68 \pm 7$ & $\ldots$. & $\ldots$. & 11 \\
$41-4810$ & $67 \pm 12$ & 1.5 & 11.5 & 16 \\
\hline
\end{tabular}

corresponds to $S\left(\tau_{\Xi}\right)$ standard deviations. $S$ is very nearly a linear function of $1 / \tau_{\Xi}$ so that the limits of $\tau_{\Xi}$ corresponding to any desired confidence level can be evaluated with a minimum of computation.

The measured values of the decay time $t_{i}$ and the gate time $T_{i}$ for each of the Caltech cascades are given in Table V. The plot of $S$ versus $1 / \tau_{\Xi}$ is shown in Fig. 1; since $S=-0.2$ for $\tau_{\Xi}=\infty$, only a lower limit to $\tau_{\Xi}$ is obtainable. To a $50 \%$ confidence level the lower limit is $4.6 \times 10^{-10} \mathrm{sec}$ while to a $95 \%$ confidence level it is $1.8 \times 10^{-10} \mathrm{sec}$.

A rough upper limit to the $\Xi^{-}$lifetime can be obtained if one assumes the validity of the prediction of the strangeness theory that every $\boldsymbol{\Xi}^{-}$is produced in association with two $K$ mesons. Thus, from knowledge of the number of $\theta^{0}$ decays observed in the chambers one can set an upper limit to the number of nondecaying particles going through the chambers and, hence, an upper limit to the lifetime of the $\Xi^{-}$. For the purposes of this analysis, it was assumed that one-half of all $K$ particles produced in association with $\boldsymbol{\Xi}^{-}$'s are neutral, and that one-half of all neutral $K$ particles decay by the usual $\theta^{0}$ mode. It was also assumed that the $\Lambda^{0}$ secondary of the $\mathbb{E}-$ always decays into charged particles; this assumption gives the most conservative upper limit. These considerations lead to an upper limit of about 800 for the number of nondecaying $z^{-}$'s, implying an upper limit in the $\boldsymbol{z}^{-}$lifetime,

$$
\tau_{\Xi}<\sim 2 \times 10^{-8} \text { sec. }
$$

\section{Angular Correlations of Decay Products of $\Xi^{-}$Events}

Gatto $^{15}$ has suggested that a useful method of obtaining information on the spin and parity of the $\boldsymbol{\Xi}^{-}$ and the $\Lambda^{0}$ is to study the distribution of the angle $\Theta$ between the $\Lambda^{0}$ direction in the rest system of the $\Xi^{-}$ and the proton direction in the rest system of the $\Lambda^{0}$. In particular, any significant departure from isotropy would imply a $\Lambda^{0}$ spin greater than $\frac{1}{2}$. Specifically, the distribution for $\Lambda^{0}$ spin $\frac{3}{2}$ would be of the form,

$$
F(\cos \theta)=1+\lambda \cos ^{2} \theta,
$$

where, in the special case where the $z^{-}$spin is $\frac{1}{2}$, the value of $\lambda$ is 3 . Values of $\theta$ for the events for which

\footnotetext{
${ }^{15}$ R. Gatto, Nuovo cimento 2, 841 (1955).
}

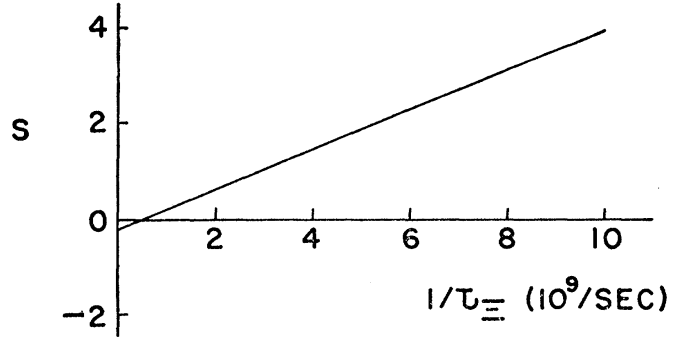

FIG. 1. Plot of $S(\tau \Xi)$ as a function of $1 / \tau_{\Xi}$.

sufficient data were available to make the calculation are shown in Table $\mathrm{V}$. These values are accurate to about $5^{\circ}$. A maximum likehood procedure was used to compute the best experimental value of $\lambda$ with the result,

$$
\lambda=2.0_{-1.3}^{+3.1}
$$

where the quoted errors are $50 \%$ confidence limits. In particular, the probability of obtaining the observed values of $\theta$ if the true value of $\lambda$ is 0 corresponds to 1.1 standard deviations or about $27 \%$. Thus, although a value of $\lambda>0$ seems to be suggested, the statistics are quite insufficient to rule out that $\lambda$ can be 0 . If the data on ${ }^{-}$decay obtained in other laboratories are combined with those presented here, more significant conclusions might be obtained.

\section{IV. $V^{-}$DECAYS EXCLUDING $K^{\prime}$ 'S AND $\Xi^{-9}$ S}

\section{A. General Discussion}

All groups of events heretofore discussed in this paper have been pure samples of some particular type of particle. This is not true, however, of the great number of unclassified $V^{-}$decays which may be expected to consist of fast $K^{-}$mesons, $\Xi^{-}$hyperons whose $\Lambda^{0}$ secondaries either decay into neutral particles or decay outside the chambers, and $\Sigma^{-}$hyperons. A $\Sigma^{-}$particle can seldom be identified positively because its short lifetime does not permit accurate measurements of its momentum. In order to determine whether appreciable numbers of these particles were present amongst the unclassified $V^{-}$events, a procedure was used whereby the numbers of unidentified $K^{-}$mesons and $\Xi^{-}$hyperons were estimated and subtracted statistically from the sample, and the properties of the remaining events were studied to see whether they correspond to the known properties of $\Sigma^{-}$particles.

\section{B. Lifetime Analysis of $V^{-}$Events}

A sample of $V^{-}$events suitable for lifetime analysis was selected and values of the decay distance, $x$, and the gate distance, $D$, were obtained for each event in the sample. In selecting the $V^{-}$events for the lifetime sample, all events with decay angles less than $10^{\circ}$ and all events whose primaries were visible in a chamber other than the one in which their decays took place were 
excluded. This last requirement was used to minimize the number of $K^{-}$and $\Xi^{-}$events in the sample. A group of $57 \mathrm{~V}^{-}$events satisfied all the requirements for the lifetime analysis.

The velocity of each of these events was computed in one of the following ways:

1. If the primary was heavily ionizing, its velocity was computed from the observed ionization.

2. If the primary was not heavily ionizing but measurements of the secondary momentum and decay angle were available, the velocity was computed from these data on the assumption of a $P^{*}$ value of 200 $\mathrm{Mev} / c$. This value is correct for the $\Sigma^{-}$'s, roughly right for the $K^{-}$'s, and wrong for the $\Xi^{-}$'s; consequently a velocity correction term was included in the $\Xi^{-}$ correction.

3. If only the decay angle was measurable, the velocity was estimated by using an empirical curve of velocity versus angle obtained from those events in group 2 above, which were sufficiently measurable to give an unbiased calibration.

The amount of $\Xi^{-}$contamination in the $V^{-}$sample was estimated from the number of identified $\Xi^{-}$- events on the assumption that the $\Lambda^{0}$ always decays into charged secondaries. On this assumption about $15 \%$ of the $V^{-}$events should have been $\Xi^{-}$'s unaccompanied by visible $\Lambda^{0}$ decays.

An estimate of the $K^{-}$contamination was made by comparing the number of identified $K^{-}$mesons with the number of $V^{-}$particles whose velocity and location in the chamber were such that had they been $K^{-}$'s and had they lived long enough, they could have been identified correctly. The results of this comparison, expressed in terms of the $\gamma \beta$ values of the primaries, are the following:

1. For $\gamma \beta<1$, roughly a third of the unidentified $V^{-}$ events are $K^{-}$particles.

2. For $1 \leqslant \gamma \beta \leqslant 2$, less than a tenth of the unidentified $V^{-}$cases are $K^{-}$particles.

The large decrease in the fraction of $K^{-}$particles with the increasing $\gamma \beta$ is entirely reasonable in view of

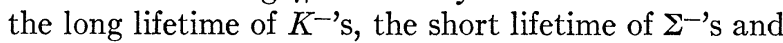
the cloud chamber geometry. These same factors suggest that the proportion of $K^{-}$'s among the unidentified $V^{-}$'s decreases even more for $\gamma \beta>2$, although this cannot be checked experimentally because one cannot separate $\Sigma$ 's from $K$ 's at such high velocities. Hence, it is advantageous, from the point of view of reducing $K^{-}$contamination, to eliminate from the lifetime sample events for which $\gamma \beta<1$.

The lifetime analysis was performed by computing the $S$ function, already discussed in connection with the $\mathbf{Z}^{-}$'s, for various assumed values of the lifetime $\tau$. The best lifetime value is given by

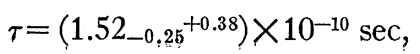

where the quoted errors, which reflect statistical and measurement uncertainties, are $50 \%$ confidence limits. This figure includes a $15 \%$ correction for $\Xi^{-}$'s and a $2 \%$ correction for $K^{-}$'s not removed by the elimination of events for which $\gamma \beta<1$.

The previous assumption concerning the absence of neutral decay modes for $\Lambda^{0}$ 's coupled with the lower limit found for $\tau_{\Xi}$, plus the fact that no correction was made for the presence of $K$ particles with $\gamma \beta>2$, require that the above value of $\tau$ be interpreted as an upper limit to the lifetime of those $V^{-}$particles which are neither $K^{-}$'s nor $\Xi^{-}$'s. If the estimates of contamination were approximately correct, this upper limit is, of course, a good approximation to the actual value.

\section{Transverse Momentum Distribution of $V$ - Events}

Information on the dynamics of the $V^{-}$events was obtained from a study of the distribution of transverse momenta $\left(P_{T}\right)$ of a sample of events especially selected to minimize the effects of unidentified $\Xi^{-}$'s and $K^{- \text {'s }}$. $\Xi^{-}$'s were completely eliminated by considering only transverse momenta above $150 \mathrm{Mev} / c$, and essentially all $K^{-}$'s were removed by including only events with $\gamma \beta>1$ whose decay occurred less than $3 \times 10^{-10}$ sec after they had left the production layer above the chamber.

The mean $P^{*}$ value for the remaining group of events was obtained from the measured transverse momenta by an averaging procedure similar to the one described in an earlier report, ${ }^{2}$ great care being used to eliminate biases. The result so obtained from a study of 19 events is

$$
P^{*}=(197 \pm 7) \mathrm{Mev} / c,
$$

where the quoted error is the probable error. It is estimated that the possible presence of $K^{-}$'s in this sample can cause this value to be too high by at most $2 \mathrm{Mev} / c$.

\section{D. $P^{*}$ Distribution of $V^{-}$Events}

Table VI gives data on 6 events whose $P^{*}$ values were obtained from knowledge of the secondary momenta and primary ionizations, but for which no measurements of primary momenta were possible. It is estimated from the number of identified heavily ionizing $K^{-}$ particles that roughly 3 unidentified $K^{-}$'s should occur. Thus the 6 events under consideration are probably not all $K^{-}$'s. The $P^{*}$ values obtained from these events are in excellent agreement with the result of the $P_{T}$ distribution analysis presented above, and are also consistent with the $P^{*}$ value of the $K_{\pi 2}$ - decay mode. In view of the impurity of the sample, the calculation of any average value is meaningless.

\section{E. Identification of $V^{-}$Events}

From the analysis given in Secs. IVB and IVC the main properties of that part of the $V^{-}$sample which is 
TABLE VI. Data on $6 V^{-}$events for which an accurate $P^{*}$ value can be obtained.

\begin{tabular}{clcccccc}
\hline \hline $\begin{array}{c}\text { Case } \\
\text { No. }\end{array}$ & \multicolumn{1}{c}{$I_{1}$} & $\begin{array}{c}P_{2} \\
(\mathrm{Mev} / c)\end{array}$ & $I_{2}$ & $\begin{array}{c}\theta \\
(\mathrm{deg})\end{array}$ & $\begin{array}{c}P^{*}(\mu) \\
(\mathrm{Mev} / c)\end{array}$ & $\begin{array}{c}P^{*}(\pi) \\
(\mathrm{Mev} / c)\end{array}$ & $\begin{array}{c}\Delta P^{*} \\
(\mathrm{Mev} / c)\end{array}$ \\
\hline 17501 & $1.5-3$ & $225 \pm 50$ & $<2$ & 36 & 132 & 132 & \pm 31 \\
34082 & $1.3-2.5$ & $186 \pm 18$ & $<2$ & 61.5 & 188 & 196 & \pm 27 \\
35487 & $2.5-5$ & $215 \pm 5$ & $<2$ & 66 & 198 & 198 & \pm 12 \\
54963 & $2-4$ & $174 \pm 7$ & $1.3-2$ & 82 & 200 & 206 & \pm 19 \\
58343 & $1.3-2$ & $153 \pm 5$ & $1.3-2$ & 82 & 204 & 218 & \pm 26 \\
64552 & $2.5-5$ & $162_{-29}^{+46}$ & $<2$ & 95 & 198 & 203 & -39 \\
& & & & & & & \\
\hline \hline
\end{tabular}

neither $K^{-}$nor $\Xi^{-}$are :

$$
\begin{aligned}
& \tau \leqslant\left(1.52_{-0.25}{ }^{+0.38}\right) \times 10^{-10} \mathrm{sec}, \\
& P^{*}=(197 \pm 7) \mathrm{Mev} / c .
\end{aligned}
$$

These properties agree quite satisfactorily with what is now known about the $\Sigma^{-}$decay,

$$
\Sigma^{-} \rightarrow n+\pi^{-} \text {. }
$$

The $Q$ value for this decay computed from the above $P^{*}$ value is $122 \pm 7 \mathrm{Mev}$. Although this value is a little higher than the known $\Sigma^{+} Q$-value, $110 \pm 1 \mathrm{Mev}{ }^{7}$ this difference is not sufficiently significant as compared to the error of measurement to constitute evidence for a mass difference between the $\Sigma^{-}$and the $\Sigma^{+}$.

If the above lifetime and $Q$ value are compared with recent measurements reported by Steinberger, ${ }^{16}$

$$
\begin{aligned}
& \tau=\left(1.4_{-0.5}^{+1.6}\right) \times 10^{-10} \mathrm{sec}, \\
& Q=118 \pm 2.6 \mathrm{Mev},
\end{aligned}
$$

the agreement is seen to be excellent. It can therefore be concluded that those $V^{-}$events which are neither $K^{-}$'s nor $\Xi^{-}$'s can be well accounted for as $\Sigma^{-}$'s.

\section{F. Neutral Decay Mode of $\boldsymbol{\Lambda}^{0}$ Particles}

It was suggested very early that the $\Lambda^{0}$ and $\theta^{0}$ particles may have alternate modes of decay,

$$
\begin{gathered}
\Lambda^{0} \rightarrow n+\pi^{0}, \\
\theta^{0} \rightarrow \pi^{0}+\pi^{0},
\end{gathered}
$$

which are not directly detectable in a cloud chamber. The fraction of $\Lambda^{0}$ particles decaying by the neutral mode can be calculated if the ratio of $\mathbf{E}^{-}$decays with an observed $\Lambda^{0}$ decay to $\Xi^{-}$decays without an observed $\Lambda^{0}$ decay is known.

Let $\rho_{\Lambda}$ be the fraction of $\Lambda^{0}$ particles which decay into charged secondaries, $n$ be the number of $\boldsymbol{\Xi}^{-}$decays for which the secondary $\Lambda^{0}$ is also observed to decay, and $N$ be the total number of $\Xi^{-}$decays observed in the cloud chamber. Define $P$ to be the mean probability for decay of the secondary $\Lambda^{0}$ inside the chamber, the average being taken over all $\Xi^{-}$decays. An estimate

${ }_{16} \mathrm{~J}$. Steinberger, Proceedings of the Sixth Annual Rochester Conference on High-Energy Physics, 1956 (Interscience Publishers, Inc., New York, to be published). of $\rho_{\Lambda}$ can be obtained from the relation

$$
\rho_{\Lambda}=n / N P \text {. }
$$

Unfortunately, because of the difficulty of identifying a $\Xi^{-}$unless the $\Lambda^{0}$ is also seen to decay, one cannot determine $N$ accurately and thus cannot use the above expression to obtain a value of $\rho_{\Lambda}$. In practice, however, one can determine an upper limit to $N$ and thus a lower limit to $\rho_{\Lambda}$.

The upper limit to $N$ was found in the following way:

1. The number $N_{1}$ of $V^{-}$and identified $\Xi^{-}$events fulfilling the qualifications required in the lifetime analysis discussed above was determined.

2. The number $N_{2}$ of $V^{-}$events included in $N_{1}$ whose $P^{*}$ or $P_{T}$ values are such as to make them inconsistent with the $\Xi^{-} P^{*}$ of $138 \mathrm{Mev} / c$ was found.

3. From the number of $V^{-}$events with $P_{T}>138$ $\mathrm{Mev} / c$, the number $N_{3}$ of events having $P^{*}>138$ $\mathrm{Mev} / c$, but $P_{T}<138 \mathrm{Mev} / c$ was obtained, using the theoretical $P_{T}$ distribution.

4. An upper limit $N^{\prime}$ to the total number of $\mathbf{Z}^{-}$'s was calculated by taking $N^{\prime}=N_{1}-N_{2}-N_{3}$.

It must be emphasized that the number $N^{\prime}$ is not an actual estimate of $N$, but is really a conservative upper limit. This is evident from the following two observations :

(i) All events of unmeasurable $P_{T}$ whether they be $\Sigma^{-}$'s, $K^{-}$'s, or $\boldsymbol{\Xi}^{-}$'s are included in $N^{\prime}$; in fact, they constitute its major part.

(ii) The number $N_{3}$ obtained as described above is really a lower limit to the number of $K^{-}$and $\Sigma^{-}$events with $P_{T}<138 \mathrm{Mev} / c$. This follows from the fact that the $P_{T}$ distribution of observed events is biased toward low values by the requirements of measurability.

The value of $P$ was computed from the decay probabilities, $P_{i}$, of the $\Lambda^{0}$ 's of the identified $\Xi^{-}$events by the relation

$$
\frac{1}{P}=\frac{1}{n} \sum_{i=1}^{n} \frac{1}{P_{i}}
$$

This formula is good only if $P_{i}$ is not allowed to have too small values. For this reason all events having $P_{i}<0.2$ were eliminated from the sample under consideration. 
TABLE VII. Data on probable $\boldsymbol{\Xi}^{-}$event.

\begin{tabular}{|c|c|c|c|c|c|c|}
\hline Particle & Sign & $\begin{array}{c}P \\
(\mathrm{Mev} / c)\end{array}$ & $I$ & $\underset{(\mathrm{cm})}{\Delta x}$ & $\begin{array}{c}\Delta y \\
(\mathrm{~cm})\end{array}$ & $\underset{(\mathrm{cm})}{\Delta z}$ \\
\hline $\begin{array}{l}\text { Primary (1) } \\
\text { Secondary (2) } \\
\text { Secondary (3) } \\
\text { Secondary (4) }\end{array}$ & $\begin{array}{l}\bar{z} \\
\bar{t}\end{array}$ & $\begin{array}{c}211 \pm 9 \\
586 \pm 48 \\
1342-250^{+400}\end{array}$ & $\begin{array}{l}<2 \\
<2 \\
<2 \\
<2\end{array}$ & $\begin{array}{r}-2.15 \\
4.13 \\
-12.64 \\
-10.67\end{array}$ & $\begin{array}{l}-7.31 \\
-23.28 \\
-33.26 \\
-31.57\end{array}$ & $\begin{array}{l}-1.55 \\
-2.64 \\
-5.34 \\
-9.96\end{array}$ \\
\hline
\end{tabular}

The final results of the analysis are the following,

$$
\rho_{\Lambda}>0.40_{-0.11^{+0.15}}
$$

where the quoted errors define a $50 \%$ confidence interval for the lower limit to $\rho_{\Lambda}$. Again it must be emphasized that only a conservative lower limit to $\rho_{\Lambda}$ and not an actual estimate has been obtained. Indeed, insofar as these data are concerned, the actual value of $\rho_{\Lambda}$ could easily be as high as unity.

\section{DESCRIPTION OF AN UNUSUAL EVENT}

In a curious event obtained during the course of this experiment, a negative particle appears to decay into three charged secondaries whose measured momenta and directions are completely incompatible with $\tau$ decay. The measured data for this event are given in Table VII. To these data may be added the fact that particle 3 cannot have been an electron because it traverses about 10 radiation lengths in lead without producing a cascade shower. The angle between the primary track and the vector sum of the secondary momenta is $2.2^{\circ} \pm 1.6^{\circ}$. This result implies that no neutral secondary need be assumed, although one may be present and may carry off a transverse momentum as high as $150 \mathrm{Mev} / c$.

A number of possible interpretations of this event immediately suggest themselves:

1. The most obvious of these is the $\tau^{-}$decay scheme,

$$
\tau^{-} \rightarrow 2 \pi^{-}+\pi^{+} \text {. }
$$

However, the $Q$ value of this event computed for the above decay scheme is $222_{-35}+56 \mathrm{Mev}$, incompatible with the known $\tau Q$ value of $75 \mathrm{Mev}$. It must be noted that the above errors are based upon the measured values of the momenta and do not give a true measure of the unlikelihood that the event is really a $\tau$ decay. A detailed analysis was made to determine what changes in the measured values of the momenta are necessary to shift the $Q$ value to $75 \mathrm{Mev}$. From this analysis, it is concluded that the interpretation of the event as an ordinary $\tau$ decay is completely ruled out.

2. One may consider the possibility that the event is some alternate decay mode of a $\tau$ meson. The following schemes were considered:

$$
\begin{aligned}
\tau & \rightarrow \pi+\pi+\mu+\nu, \\
& \rightarrow \pi+\pi+e+\nu, \\
& \rightarrow \pi+\mu+e .
\end{aligned}
$$

Only schemes (ii) and (iii) were compatible with the data. In view of the fact that out of hundreds of ob- served $\tau^{+}$decays, no examples of the above schemes have been detected, it is believed very unlikely that the event really represents an alternate decay mode of a $\tau^{-}$.

3. The event may be interpreted as a nuclear interaction in the gas of the cloud chamber. However, the absence of any visible recoil blob and of evaporation protons makes this interpretation unlikely. Furthermore, although the good transverse momentum balance suggests that no neutral particle is emitted, if this event is assumed to be a nuclear interaction, it is impossible to conserve momentum and energy using only the visible particles and the assumed target nucleon.

4. The event may be a $\mathbf{Z}^{-}-\Lambda^{0}$ cascade decay in which the $\Lambda^{0}$ decays very rapidly after being created. The validity of this interpretation may be tested by a study of the consistency of the measured data with the $\Xi^{-}$and $\Lambda^{0}$ dynamics. In particular, if particles 2 and 4 are assumed to be the $\Lambda^{0}$ secondaries, the $\Lambda^{0} Q$ value comes out $35_{-5}^{+8} \mathrm{Mev}$, and the $\Xi^{-} Q$ value comes out $75_{-28}{ }^{+20} \mathrm{Mev}$, both in satisfactory agreement with the accepted values of these quantities. Furthermore, if the momentum of the assumed proton is calculated from the known $\Lambda^{0} Q$-value (37 Mev) and the accurately measured momentum of particle 2, the $\boldsymbol{\Xi}^{-} Q$ value then obtained is $71 \pm 12 \mathrm{Mev}$. These results strongly support this interpretation of the event.

It is estimated that the $\Lambda^{0}$ must decay within $1 \mathrm{~mm}$ of the $\Xi^{-}$-apex implying a time of flight of $2 \times 10^{-12} \mathrm{sec}$. The probability that the time of flight of the $\Lambda^{0}$ in this particular event is as short or shorter than this value is about $0.7 \%$. On the other hand if the other $\Xi^{-}$'s obtained are considered, the chance that one of them has such a short-lived $\Lambda^{0}$ is about $8 \%$, which is appreciable.

On the basis of the above arguments, it is believed that the event may be most easily interpreted as a $\Xi^{-}-\Lambda^{0}$ decay. This case has not been included in the $\Xi^{-}$ sample previously discussed because the uncertainties in its identification are greater than was the case for the other $\Xi^{-}$events. If, however, the above identification is accepted as correct, and the case is included with the other $\Xi^{-}$events, the values of $Q_{\Xi}, \tau_{\Xi}, \lambda$, and $\rho_{\Lambda}$ thereby obtained are the following:

$$
\begin{aligned}
Q_{\Xi} & =65 \pm 3 \mathrm{Mev}, \\
\tau_{\Xi} & <3.1 \times 10^{-10} \mathrm{sec},(50 \% \text { confidence limit }) \\
\lambda & =1.8_{-1.3}+2.3 \\
\rho_{\Lambda} & =0.43_{-0.10}+0.14
\end{aligned}
$$

\section{ACKNOWLEDGMENTS}

The authors wish to express their appreciation to Professor C. D. Anderson and Professor R. B. Leighton for constant encouragement and valuable discussions. They are also indebted to Dr. V. A. J. van Lint, Dr. A. A. Strassenburg, Dr. C. A. Rouse, Dr. J. D. Sorrels, J. A. Kadyk, and R. L. Luttermoser for assistance in the operation of the equipment and the analysis of the data. 